\title{
Involvement of calpain in colorectal adenocarcinomas (Review)
}

\author{
PONNIAH SELVAKUMAR and RAJENDRA K. SHARMA
}

\begin{abstract}
Department of Pathology and Laboratory Medicine, College of Medicine, University of Saskatchewan, and Cancer Research Unit, Saskatoon Cancer Center, University of Saskatchewan, Saskatoon, Saskatchewan S7N 4H4, Canada
\end{abstract}

Received March 5, 2010; Accepted March 22, 2010

DOI: 10.3892/etm_00000064

\begin{abstract}
Calpains represent a well-conserved family of $\mathrm{Ca}^{2+}-$ dependent proteolytic enzymes. Recently, the importance of calpain in the metastatic process has received a great deal of attention. Various reports have suggested that $m$-calpain contributes to the pathogenesis of various cancers, including colorectal cancer. The activity and protein expression of $m$-calpain was significantly higher in colorectal adenocarcinoma than in normal colonic mucosa as revealed by Western blotting and immunohistochemical analysis. In addition, the decreased expression of calpain inhibitors (calpastatin and high molecular weight calmodulin-binding protein) was correlated with the increased activity and expression of calpain in colorectal adenocarcinoma. This has implications with regard to the design of chemotherapeutic drugs as well as for the monitoring of colorectal cancer in the early stages of the metastatic process. In this review, we summarize some of the recent findings from our laboratory regarding calpain and its inhibitors in human colon cancer.
\end{abstract}

\section{Contents}

1. Introduction

2. The calpain family

3. Calpain in pathophysiology

4. Calpain in colon cancer

5. Biological inhibitors of calpain

6. Conclusion

\section{Introduction}

Calpain is an intracellular $\mathrm{Ca}^{2+}$-dependent cysteine protease that is ubiquitously distributed throughout the body. Calpain plays a significant role in various cellular functions such as

Correspondence to: Dr Rajendra K. Sharma, Department of Pathology and Laboratory Medicine, College of Medicine, University of Saskatchewan, and Cancer Research Unit, Saskatoon Cancer Center, Saskatoon, Saskatchewan S7N 4H4, Canada

E-mail: rajendra.sharma@saskcancer.ca

Key words: calpain, cancer, therapeutics, biomarkers signal transductions and cell morphogenesis $(1,2)$. Various reports have suggested that humans have 15 genes that encode a calpain-like protease domain. These generate diverse types of calpain homologues with combinations of several functional domains, such as $\mathrm{Ca}^{2+}$-binding and the $\mathrm{Zn}$-finger domains $(1,2)$. The purpose of this review is to summarize the cross-talk between $m$-calpain and its inhibitors, calpastatin and high molecular weight calmodulin-binding protein (HMWCaMBP), in human colorectal cancer.

\section{The calpain family}

Calpains are $\mathrm{Ca}^{2+}$-activated cysteine proteases that act as major mediators for $\mathrm{Ca}^{2+}$ signals in many biological systems $(1,2)$. There are two types of calpains, I or $\mu$ - and II or $m$-calpain, which require a micromolar and millimolar concentration of $\mathrm{Ca}^{2+}$ for activation, respectively $(1,2)$. Both calpains are heterodimers consisting of a common small subunit $(28 \mathrm{kDa})$ with a regulatory function and a distinct large catalytic subunit (80 kDa). Different mechanisms responsible for $m$-calpain regulation have been reported, and an important role has been ascribed to the specific inhibitor calpastatin (3-5). In addition, an endogenous calpain inhibitor, HMWCaMBP, was identified and characterized in our laboratory (6). Based on sequence homology, amino acid analysis, antibody reactivity and calpain inhibition, we demonstrated that HMWCaMBP is homologous to calpastatin, an endogenous inhibitor of calpains (7).

Calpain has catalytic and regulatory subunits that can be divided into 4 and 2 domains, respectively (1-5). The $\mathrm{N}$-terminus of domain I of the large subunit is autolyzed upon activation by $\mathrm{Ca}^{2+}$ in order to have a lower $\mathrm{Ca}^{2+}$ requirement, and the autolysis results in the dissociation of the subunits. Therefore, autolysis is involved in the regulation of calpain activity and specificity (1). Three-dimensional structural studies revealed that the protease domain in the absence of $\mathrm{Ca}^{2+}$ is divided into two sub-domains, domains IIa and $\mathrm{IIb}$, which are folded into one domain upon $\mathrm{Ca}^{2+}$ binding (1-4). This domain is most conserved among calpain family members, suggesting it has indispensable functions. The protease domain of $\mu$ - and $m$-calpains without other domains showed $\mathrm{Ca}^{2+}$-dependent protease activity. This is supported by $3-\mathrm{D}$-structural studies of the protease domain in the presence of $\mathrm{Ca}^{2+}$, which showed $\mathrm{Ca}^{2+}$ binding to domains IIa and IIb. Thus, the $\mathrm{Ca}^{2+}$-dependency of calpains is controlled as a whole molecule, since all domains (IIa, IIb, III, IV and VI) bind at least one $\mathrm{Ca}^{2+}$ with different affinities (1-4). 


\section{Calpain in pathophysiology}

Recently, the importance of calpain in the metastatic process has received a great deal of attention. Calpain may be involved in cell adhesion, spreading, migration, myoblast fusion, cell cycle control and mitosis (8-10). Calpain-mediated proteolysis represents a major pathway of post-translational modification that influences various aspects of cell physiology, including apoptosis, cell migration and cell proliferation (1). Calpains cause limited proteolysis of substrates, resulting in the alteration of substrate activity. PEST sequences are believed to be the intramolecular signals for rapid proteolytic degradation by $m$-calpain. Lakshmikuttyamma et al $(11,12)$ reported that the increase in calcineurin $(\mathrm{CaN})$ activity and strong immunostaining in ischemic/perfused rat hearts may be due to the $m$-calpain-mediated proteolysis of $\mathrm{CaN}$. In addition, the interaction of $\mathrm{CaN}$ with $m$ - and $\mu$-calpains was strong in epileptic chickens compared to normal birds $(13,14)$. It has also been reported that $\mathrm{N}$-myristoyltransferase (NMT) interacts with $m$-calpain in epileptic chickens $(14,15)$. Among two forms of NMTs (NMT1 and NMT2), a higher interaction of $m$-calpain with NMT2 was observed $(14,15)$.

\section{Calpain in colon cancer}

Calpains result in the proteolysis of a broad spectrum of cellular proteins (2), including multiple signaling enzymes, protein kinase C, pp60 ${ }^{\mathrm{c}-S r c}$ and tyrosine phosphatase $1 \mathrm{~B}$ (16-18). Most of the substrate proteins of calpains have been implicated in the pathogenesis of human tumors, suggesting an important regulatory role of calpains in malignant diseases. The role of calpains in carcinogenesis and tumor progression has yet to be explored. In human renal cell carcinomas, significantly higher levels of $\mu$-calpain expression were found in tumors that had metastasized to peripheral lymph nodes compared to tumors that apparently had not metastasized (19). It has been reported that the epigenetic activation of calpain II plays an important role in the invasion of human prostate cancer and can be targeted to reduce tumor progression (20). Gastric-specific calpain-9 is down-regulated in carcinomas, and its relation to differentiation status or tumorigenesis remains unclear $(21,22)$. The activity and expression of $\mu$-calpain were significantly increased in chronic lymphocytic leukemia cells compared to non-malignant cells, whereas the activity and expression of $m$-calpain and calpastatin were unchanged (23).

The proto-oncogenes c-fos and c-jun, several cytoskeletal proteins, the tumor suppressor protein p53 and signaling molecules protein kinase $\mathrm{C}$ and focal adhesion kinase (FAK; a non-receptor kinase) are substrates for calpain (24-26). Calpain-mediated cleavage of FAK and focal adhesion disassembly accompany v-Src-induced morphological transformation (26). v-Src-induced oncogenic transformation is characterized by alterations in cell morphology, adhesion, motility, survival and proliferation $(27,28)$. In response to v-Src activation, Carragher et al (29) demonstrated an increase in the total protein levels of calpain II and decreased levels of calpastatin in chicken embryo fibroblasts. Furthermore, the data suggested a feed-back loop mechanism of calpain activation initiated in response to the activation of the $\mathrm{v}-\mathrm{Src}$ oncogene (29). Activation of Src, which has intrinsic tyrosine kinase activity, has been demonstrated in human solid tumors such as colorectal and breast carcinomas $(30,31)$. We observed that the increased activity and expression of $m$-calpain corresponded to a decrease in the expression of calpastatin and HMWCaMBP in adenocarcinoma (32). Selvakumar et al (33) reported that the protein-protein interaction of NMTs revealed that $m$-calpain interacts with NMT1, while caspase-3 interacts with NMT2 in human colorectal adenocarcinomas. Previously, our laboratory reported that $m$-calpain proteolyses NMT1 and abolishes the enzyme activity (34).

Cell death by apoptosis is a fundamental process controlling the normal development and homeostasis of multicellular organisms. Decreased apoptotic susceptibility contributes to the pathogenesis of several diseases including cancer (1-5). Calpains are involved in controlling the level and duration of transduction signals leading to either proliferation or apoptosis in multiple cell systems $(1,3)$. However, their role in the development and course of apoptosis is controversial. A central regulator of apoptotic susceptibility is the tumor suppressor protein $\mathrm{p} 53$, whose level is regulated by several stress conditions, cell adhesion and the expression of several oncogenes $(35,36)$. It has also been reported that p53 is proteolytically cleaved in vitro by calpains $(25,37)$. The elevated expression of $m$-calpain in colorectal cancer may act on p53, and is followed by a decrease in the event of apoptosis. Likewise, the calpainmediated cleavage of Bax promotes the pro-apoptotic effect of Bax (38), and the calpain cleavage of pro-caspase-7 and procaspase-3 leads to the activation of these proteases $(39,40)$. Chen et al (41) reported a reduction in the protein levels of caspase-3, -7 and -9 in human colon cancer specimens. The apoptosis promoting caspase system is activated after calpain inhibition with calpain inhibitor II in neoplastic lymphoid cells (42). Cross-talk between calpain and caspases appears to be important for the regulation of apoptosis in colon tumors.

Various reports, including those from our laboratory, have suggested that $m$-calpain is involved in the progression of metastasis $(26,32)$. In order to analyze the role of $m$-calpain in human colorectal adenocarcinoma, calpain activity and protein expression was examined in human tissue samples. In most of the cases, the calpain activity was significantly higher in human colorectal adenocarcinoma than in normal mucosa ( $83 \%$ of cases, $\mathrm{P}<0.05$ ) (Fig. 1). Notably, $m$-calpain activity was higher in polyps than normal tissues, though not as high as in cancerous tissues (Fig. 1A). Furthermore, a higher expression of $m$-calpain was found in cancerous tissues, whereas it was poorly expressed in normal mucosa as determined by Western blot analysis (Fig. 1B, panel I). Quantitative analysis of the $80-\mathrm{kDa}$ band revealed a 2- to 3-fold higher expression $(\mathrm{P}<0.05)$ of $m$-calpain in colorectal tumors compared to their respective normal mucosa (Fig. 1B, panel II). However, no change in expression was observed at the $28 \mathrm{kDa}$ small subunit of $m$-calpain (unpublished data). In polyps, the expression of $m$-calpain was higher than in normal tissues, while no significant change was observed in the remaining normal tissues (Fig. 1B, panel II).

Immunohistochemical analysis showed strong staining for $m$-calpain in colorectal adenocarcinoma (Fig. 2A-c) by the avidin-biotin complex method graded as described previously $(43,44)$. Mild reactivity $(<10 \%$ of protein expression) was observed in mucosal sections taken distant from the cancerous 

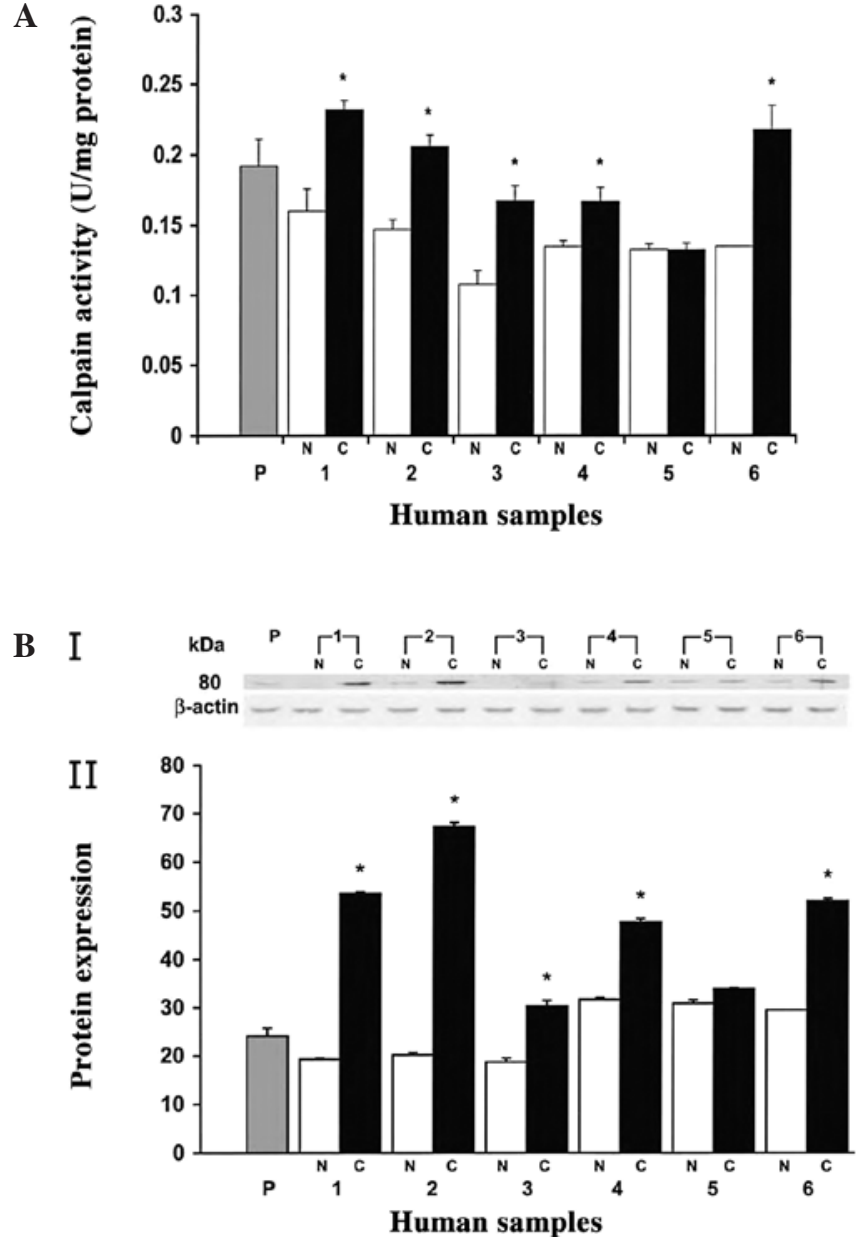

Figure 1. (A) Calpain activity of human colorectal normal ( $\square$ ), polyp ( $\square$ ) and tumor (घ) tissue. (B) Expression of $m$-calpain in human colorectal adenocarcinoma. Panel I, Western blot analysis of $m$-calpain in human colorectal

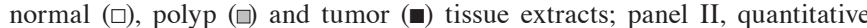
analysis of Fig. 1B, panel I (32).

tissues (Fig. 2A-a). In polyps, moderate staining was observed, and the degree of immunoreactivity was less than in tumor tissue (Fig. 2A-b). From these studies and various reports, it is clear that $m$-calpain is a major player during the process of various diseases, including cancer, cardiovascular and neurological diseases.

\section{Biological inhibitors of calpain}

Calpain activity is tightly regulated by its ubiquitously expressed endogenous inhibitor, calpastatin (3-5). We also monitored the expression of the calpain inhibitor, calpastatin, to determine whether these endogenous inhibitors regulate calpain activity in colorectal adenocarcinoma (32). Western blot analysis of calpastatin revealed strong expression in normal mucosa, whereas weak expression was observed in colorectal tumors (Fig. 2B, panel I). Quantitative analysis of calpastatin revealed an approximately 2-fold increased expression in normal tissues compared to cancerous tissues (Fig. 2B, panel II). The weak expression of calpastatin in colon tumors was further confirmed by immunohistochemical analysis (Fig. 2C-b). Moderate-to-strong staining was observed for normal mucosa, while a weak cytoplasmic positivity was
A

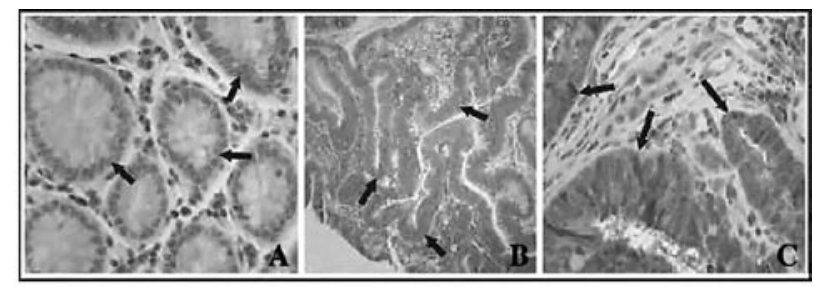

B I

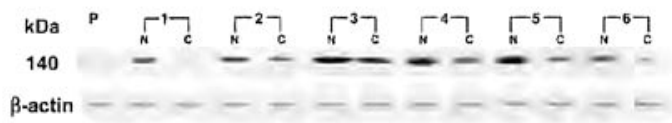

II

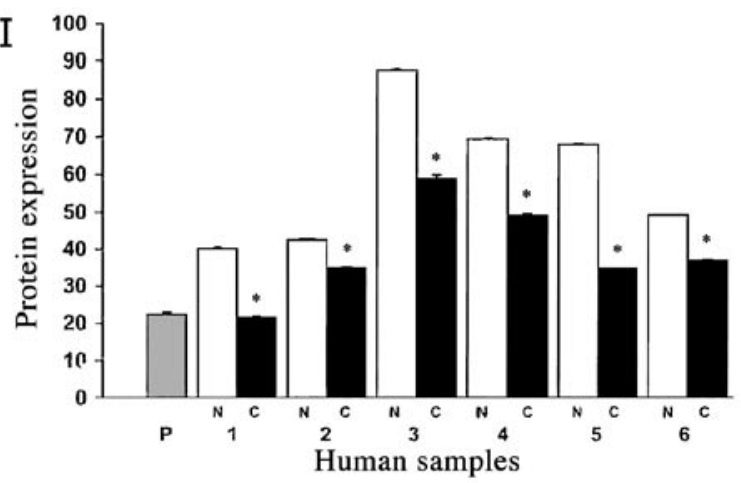

C

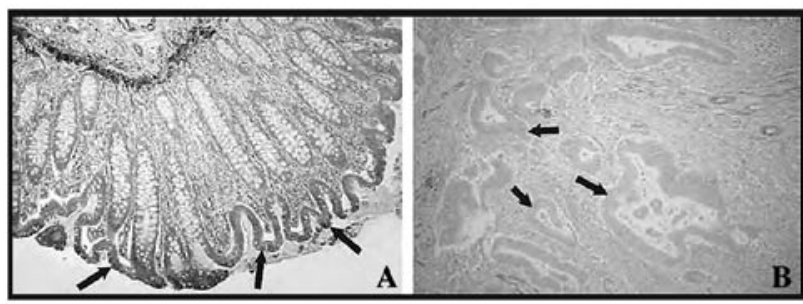

D

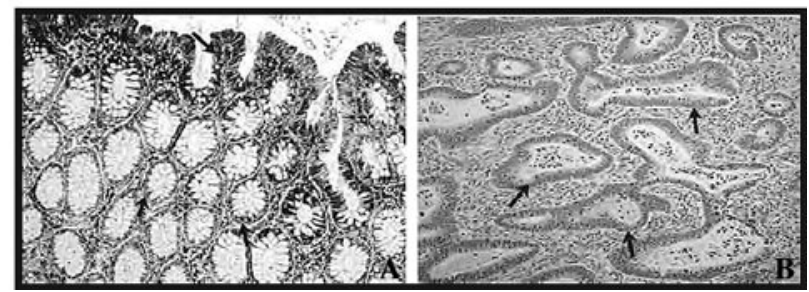

Figure 2. (A) Immunohistochemical analysis of $m$-calpain. Section from (a) normal mucosa, (b) polyps and (c) colorectal adenocarcinoma. (B) Expression of calpastatin in human colorectal adenocarcinoma. Panel I, Western blot analysis of calpastatin in human colorectal normal ( $\square$ ), polyp ( $\square$ ) and tumor ( $\square$ ) tissue extracts; panel II, quantitative analysis of Fig. 2A(a). (C) Immunohistochemical staining for calpastatin using anti-calpastatin Sections from (a) normal mucosa and (b) colorectal adenocarcinoma. (D) Immunohistochemical staining for HMWCaMBP using anti-HMWCaMBP Sections from (a) normal mucosa and (b) colorectal adenocarcinoma (arrows, immunoperoxidase; original magnification, x120).

observed for calpastatin in invasive carcinoma, with decreased intensity in the invasive component (Fig. 2C-a).

We also investigated the role of another calpain endogenous inhibitor, HMWCaMBP, which was identified and characterized at our laboratory in human colorectal adenocarcinomas (32). Similar to calpastatin, Western blot analysis was carried out using anti-HMWCaMBP and showed a weakly expressed immunoreactive band with an apparent molecular mass of $140 \mathrm{kDa}$ in colon tumors. Significantly higher staining with anti-HMWCaMBP was observed in normal mucosa (unpublished data). Furthermore, immunohistochemical studies 
revealed mild reactivity of HMWCaMBP in colorectal adenocarcinoma (Fig. 2D-b). However, the mucosal sections taken distant from the tumor showed strong staining (Fig. 2D-a). We observed that the increased activity and expression of $m$-calpain was correlated with the decreased expression of calpastatin and HMWCaMBP in human colorectal adenocarcinoma.

\section{Conclusion}

Apart from the known regulatory functions of calpains, various reports, including ours, suggest that increased $m$-calpain expression may directly contribute to the development of cell progression in colorectal adenocarcinoma. The determination of the mechanisms causing the increase in calpain expression and its action on cell signaling may yield data critical for addressing many unanswered questions about cell proliferation in colon tumors. Increased activity and moderate staining of $m$-calpain in polyps demonstrate the usage of this enzyme as a marker for the early detection of colorectal adenocarcinoma using immunological approaches. The overexpression of calpastatin or HMWCaMBP, which are specific for calpain inhibition, may be used as a specific molecular target for the treatment of colon cancer.

\section{Acknowledgements}

This study was supported by the Canadian Institutes of Health Research, Canada. The authors are thankful to Dr J.R. Dimmock, College of Pharmacy and Nutrition, University of Saskatchewan, for the critical reading of the manuscript and suggestions, and to Mr. Mark F. Boyd and Mr. Todd Reichert for the technical and photographic work.

\section{References}

1. Sorimachi H, Ishiura S and Suzuki K: Structure and physiological function of calpains. Biochem J 328: 721-732, 1997.

2. Goll DE, Thompson VF, Li H, Wei W and Cong J: The calpain system. Physiol Rev 83: 731-801, 2002.

3. Ono Y, Sorimachi H and Suzuki K: Structure and physiology of calpain, an enigmatic protease. Biochem Biophys Res Commun 245: 289-294, 1998.

4. Maki M, Ma H, Takano E, Adachi Y, Lee WJ, Hatanaka M and Murachi T: Calpastatins: biochemical and molecular biological studies. Biomed Biochim Acta 50: 509-516, 1991.

5. Carragher NO: Calpain inhibition: a therapeutic strategy targeting multiple disease states. Curr Pharm Des 12: 615-638, 2006.

6. Sharma RK: Purification and characterization of novel calmodulin-binding protein from cardiac muscle. J Biol Chem 265: 1152-1157, 1990.

7. Kakkar R, Raju RV, Mellgren RL, Radhi J and Sharma RK: Cardiac high molecular weight calmodulin binding protein contains calpastatin activity. Biochemistry 36: 11550-11555, 1997.

8. Carragher NO and Frame MC: Calpain: a role in cell transformation and migration. Int J Biochem Cell Biol 34: 1539-1543, 2002.

9. Huttenlocher A, Palecek SP, Lu Q, Zhang W, Mellgren RL, Lauffenburger DA, Ginsberg MH and Horwitz AF: Regulation of cell migration by the calcium-dependent protease calpain. J Biol Chem 272: 32719-32722, 1997.

10. Patel YM and Lane MD: Mitotic clonal expansion during preadipocyte differentiation: calpain-mediated turnover of p27. J Biol Chem 275: 17653-17660, 2000.

11. Lakshmikuttyamma A, Selvakumar P, Kakkar R, Kanthan R, Wang R and Sharma RK: Activation of calcineurin expression in ischemia-reperfused rat heart and in human ischemic myocardium. J Cell Biochem 90: 987-997, 2003.
12. Lakshmikuttyamma A, Selvakumar P, Sharma AR, Anderson DH and Sharma RK: In vitro proteolytic degradation of bovine brain calcineurin by m-calpain. Neurochem Res 29: 1913-1921, 2004.

13. Lakshmikuttyamma A, Selvakumar P, Charavaryamath C, Singh B, Tuchek J and Sharma RK: Expression of calcineurin and its interacting proteins in epileptic fowl. J Neurochem 96 : 366-373, 2006.

14. Lakshmikuttyamma A, Selvakumar P, Tuchek J and Sharma RK: Myristoyltransferase and calcineurin: novel molecular therapeutic target for epilepsy. Prog Neurobiol 84: 77-84, 2008.

15. Selvakumar $P$, Lakshmikuttyamma A, Charavaryamath $C$, Singh B, Tuchek J and Sharma RK: Expression of myristoyltransferase and its interacting proteins in epilepsy. Biochem Biophys Res Commun 335: 1132-1139, 2005.

16. Kishimoto A, Kajikawa N, Shiota M and Nishizuka Y: Preoteolytic activation of calcium-activated, phospholipiddependent protein kinase by calcium-dependent neutral protease. J Biol Chem 258: 1156-1164, 1983.

17. Oda A, Drucker BJ, Ariyoshi H, Smith M and Salzman E: pp60 $0^{\text {src }}$ is an andogenous substrate for calpain in human blood platelets. J Biol Chem 268: 12603-12608, 1993.

18. Frangioni JV, Oda A, Smith M, Salzman EW and Neel BG: Calpain-catalyzed cleavage and subcellular relocation of protein phosphotyrosine phosphatase 1B PTB-1B in human platelets. EMBO J 12: 4843-4856, 1993.

19. Braun C, Engel M, Seifert M, Theisinger B, Seitz G, Zang KD and Welter C: Expression of calpain I messenger RNA in human renal cell carcinoma: correlation with lymph node metastasis and histological type. Int J Cancer 84: 6-9, 1999.

20. Mamoune A, Luo JH, Lauffenburger DA and Wells A: Calpain-2 is a target for limiting prostate cancer invasion. Cancer Res 63: 4632-4640, 2003.

21. Yoshikawa Y, Mukai H, Hino F, Asada K and Kato I: Isolation of two novel genes, down regulated in gastric cancer. Jpn J Cancer Res 91: 459-463, 2000.

22. Liu K, Li L and Cohen SN: Antisense RNA-mediated deficiency of the calpain protease, $\mathrm{nCL}-4$, in NH3T3cells is associated with neoplastic transformation and tumorigenesis. J Biol Chem 275: 31093-31098, 2000.

23. Witkowski JM, Zmuda-Trzebiatowska E, Swiercz JM, Cichorek M, Ciepluch H, Lewandowski K, Bryl E and Hellmann A: Modulation of the activity of calcium-activated neutral proteases calpains in chronic lymphocytic leukemia B-CLL cells. Blood 100: 1802-1809, 2002.

24. Carillo S, Pariat M, Steff AM, Roux P, Etienne-Julan M, Lorca T and Piechaczyk M: Differential sensitivity of FOS and JUN family members to calpains. Oncogene 9: 1679-1689, 1994.

25. Kubbutat MH and Vousden KH: Proteolytic cleavage of human p53 by calpain: a potential regulator of protein stability. Mol Cell Biol 17: 460-468, 1997.

26. Carragher NO, Fincham VJ, Riley D and Frame MC: Cleavage of focal adhesion kinase by different proteases during SRC-regulated transformation and apoptosis. Distinct roles for calpain and caspases. J Biol Chem 276: 4270-4275, 2001.

27. Johnson D, Frame MC and Wyke JA: Expression of the v-Src oncoprotein in fibroblasts disrupts normal regulation of the CDK inhibitor p27 and inhibits quiescence. Oncogene 16: 2017-2028, 1998.

28. Kellie S: Cellular transformation, tyrosine kinase oncogenes and the cellular adhesion plaque. Bioessays 8: 25-30, 1988.

29. Carragher NO, Westhoff MA, Riley D, Potter DA, Dutt P, Elce JS, Greer PA and Frame MC: v-Src-induced modulation of the calpain-calpastatin proteolytic system regulates transformation. Mol Cell Biol 22: 257-269, 2002.

30. Bolen JB, Veillette A, Schwartz AM, DeSeau V and Rosen N: Activation of pp60 $0^{\mathrm{c}-\mathrm{src}}$ protein kinase activity in human colon carcinoma. Proc Natl Acad Sci USA 84: 2251-2255, 1987.

31. Ottenhoff-Kalff AE, Rijksen G, van Beurden EA, Hennipman A, Michels AA and Staal GE: Characterization of protein tyrosine kinases from human breast cancer: involvement of the c-src oncogene product. Cancer Res 52: 4773-4778, 1992.

32. Lakshmikuttyamma A, Selvakumar P, Kanthan R, Kanthan SC and Sharma RK: Overexpression of $\mathrm{m}$-calpain in human colorectal adenocarcinomas. Cancer Epidemiol Biomarkers Prev 13: 1604-1609, 2004.

33. Selvakumar P, Smith-Windsor E, Bonham K and Sharma RK: N-myristoyltransferase 2 expression in human colon cancer: cross-talk between the calpain and caspase system. FEBS Lett 580: 2021-2026, 2006. 
34. Raju RV, Kakkar R, Datla RS, Radhi J and Sharma RK: Myristoyl-coA: protein N-myristoyltransferase from bovine cardiac muscle: molecular cloning, kinetic analysis and in vitro proteolytic cleavage by m-calpain. Exp Cell Res 241: 23-35, 1998.

35. Levine AJ: p53, the cellular gatekeeper for growth and division. Cell 88: 323-331, 1997.

36. Giaccia AJ and Kastan MB: The complexity of p53 modulation: emerging patterns from divergent signals. Genes Dev 12: 2973-2983, 1998.

37. Gonen H, Shkedy D, Barnoy S, Kosower NS and Ciechanover A On the involvement of calpains in the degradation of the tumor suppressor protein p53. FEBS Lett 406: 17-22, 1997.

38. Toyota H, Yanase N, Yoshimoto T, Moriyama M, Sudo T and Mizuguchi J: Calpain-induced Bax-cleavage product is a more potent inducer of apoptotic cell death than wild-type Bax. Cancer Lett 189: 221-230, 2003.

39. Blomgren K, Zhu C, Wang X, Karlsson JO, Leverin AL, Bahr BA Mallard C and Hagberg H: Synergistic activation of caspase-3 by $\mathrm{m}$-calpain after neonatal hypoxia-ischemia: a mechanism of 'pathological apoptosis'? J Biol Chem 276: 10191-10198, 2001.
40. Ruiz-Vela A, Gonzalez de Buitrago $G$ and Martinez AC: Implication of calpain in caspase activation during B cell clonal deletion. EMBO J 18: 4988-4998, 1999.

41. Chen T, Yang I, Irby R, Shain KH, Wang HG, Quackenbush J, Coppola D, Cheng JQ and Yeatman TJ: Regulation of caspase expression and apoptosis by adenomatous polyposis coli. Cancer Res 63: 4368-4374, 2003.

42. Zhu DM and Uckun FM: Calpain inhibitor II induces caspasedependent apoptosis in human acute lymphoblastic leukemia and non-Hodgkin's lymphoma cells as well as some solid tumor cells. Clin Cancer Res 6: 2456-2463, 2000.

43. Raju RV, Moyana TN and Sharma RK: N-myristoyltransferase overexpression in human colorectal adenocarcinomas. Exp Cell Res 235: 145-154, 1997.

44. Moyana TN and Xiang J: Expression of tumor-associated polymorphic epithelial mucin and carcinoembryonic antigen in gastrointestinal carcinoid tumors. Cancer 75: 2836-2843, 1995. 